Vol.1 No.1, Spring 2020

\title{
Determinant factors of death depression in iranian patients with cancer
} Goudarzian $\mathrm{AH}^{1}$, Sharif Nia $\mathrm{H}^{2}$, Sheikh Raberi $\mathrm{A}^{3 *}$, Tahmasbi $\mathrm{M}^{4}$, Farokhmanesh $\mathrm{K}^{5}$,

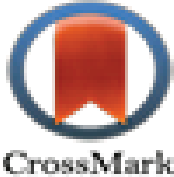

Soleimani $\mathrm{MA}^{6}$, Pour Ramzani $\mathrm{N}^{7}$

\section{CrossMark}

$\rightarrow$

Alick for updates

1- B.Sc of Nursing, Student Research Committee, Mazandaran University of Medical Sciences, Sari, Iran.

2- Ph.D. Assistant Professor, School of Nursing and Midwifery Amol, Mazandaran University of Medical Sciences, Sari, Iran.

3- M.Sc of Medical-Surgical Nursing, Sirjan University of Medical Sciences, Kerman, Iran.

4- BSc of Nursing, Imam Khomeini Hospital of Sari, Mazandaran University of Medical Sciences, Sari, Iran.

5- B.Sc of Nursing, Amol Imam Reza Hospital, Mazandaran University of Medical Sciences, Sari, Iran.

6- Ph.D. Social Determinants of Health Research Center, Qazvin University of Medical Sciences, Qazvin, Iran.

7- M.Sc of Medical-Surgical Nursing, Sirjan University of Medical Sciences, Kerman, Iran.

Corresponding Author: Sheikh Raberi A, .M.Sc of Medical-Surgical Nursing, Sirjan University of Medical Sciences, Kerman, Iran.

Email: p.shykhrabori@gmail.com

Received: 22 March 2019

Accepted: 19 April 2019

\section{Abstract}

Introduction: Living with a serious stressor such as cancer is known to activate cognitive patterns relative to personal death and dying. This study was conducted to determine of death depression and its related factors in Iranian patients with cancer.

Method: In this cross-sectional study that was conducted in 2015 (June-November); 500 cancer patients with random sampling method were included to study. Data were gathered with demographic and Death Depression Scale (DDS). Univariate and multivariate linear regression analyses with using SPSS 22.0 were used to explore the potential factors influencing death depression of cancer patients.

Results: Based on results, males age (48.59 \pm 16.15 ; 95CI: 46.62-50.57) were more than females (46.38 \pm 14.24 ; 95 CI: 68.42-71.66). The average of death depression score among patient with cancer was higher than the third quartile. A multivariate linear regression analysis showed that scores of incoming status $(\beta$ coefficient $=0.24$, $\mathrm{p}<0.001)$, no history of drug use $(\beta$ coefficient $=0.13, p=0.002)$, and stage of cancer $(\beta$ coefficient $=0.12$, $\mathrm{p}=0.005$ ) were independent predictors of death depression in cancer patients.

Conclusion: Regards to high prevalence of depression in selected patients, importance of concentration on psychology interventions, especially in acute phase of disease and chemotherapy periods were highlighted.

Keywords: Death Depression, Cancer, Related factors, Iran, Patient.

\begin{tabular}{|l|l|}
\hline \multicolumn{3}{|c|}{ Access this article online } \\
\hline Website: \\
\hline Www.ijca.ir \\
\hline
\end{tabular}




\section{فاكتور هاى مؤثر بر افسردكى مر تى در مددجويان مبتلا به سرطان}

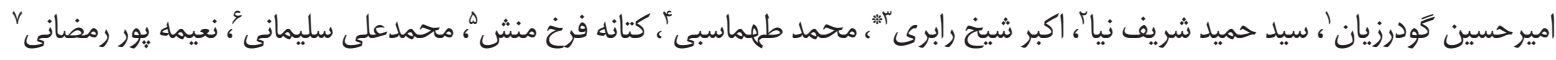

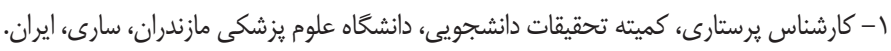

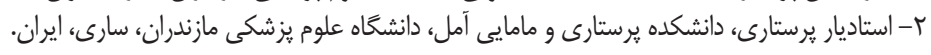

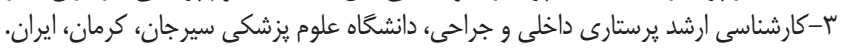

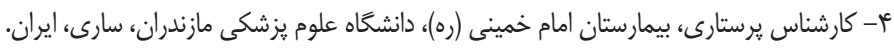

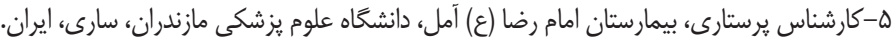

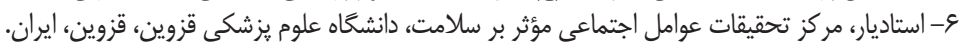

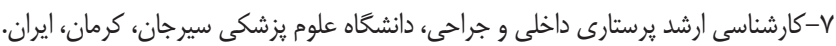

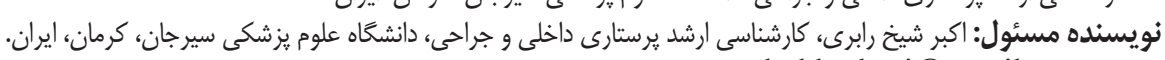
ايميل: p.shykhrabori@gmail.com

\section{Or.}

مقدمه: زندكَى با يك عامل تنشزاى جدى مانند سرطان، به عنوان يك الكَوى شناخته شده فعال مرتبط با مركَ و مردن شناخته شده است.

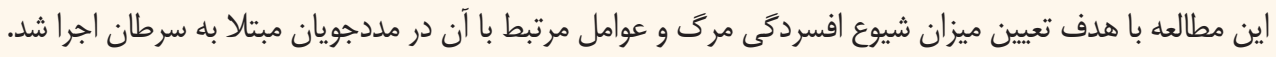

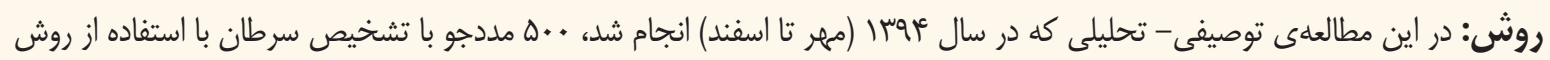

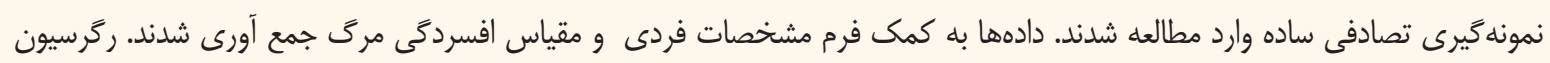

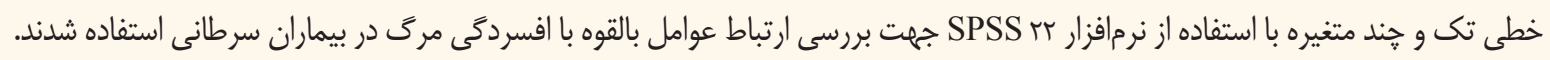

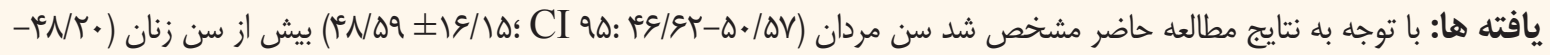

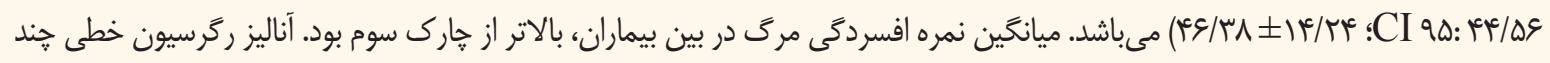

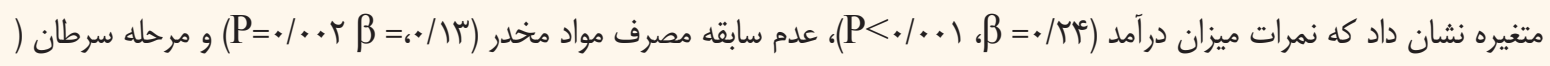

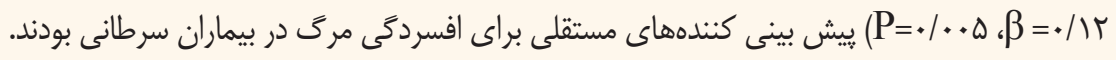

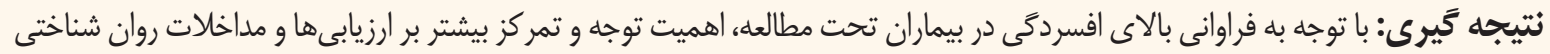

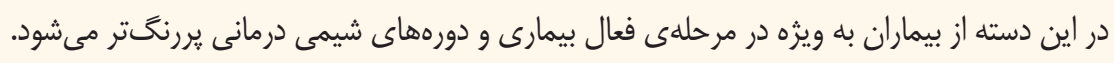
كليد وازه ها: افسردگى مرگى، سرطان، عوامل مرتبط، بيمار. 


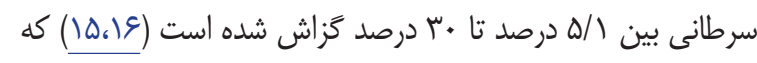

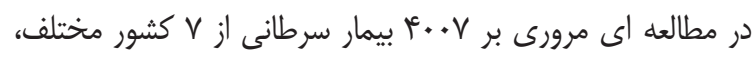

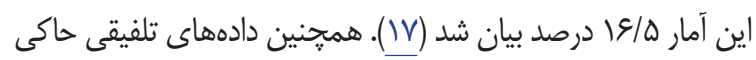

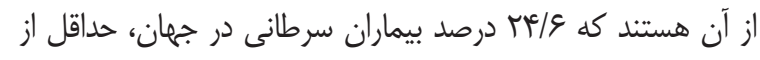

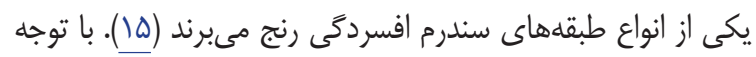

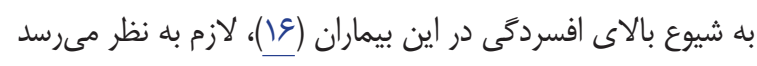

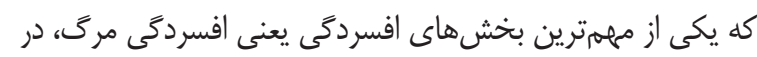

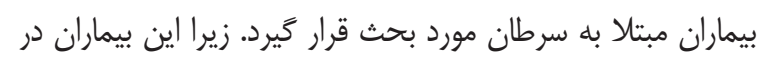

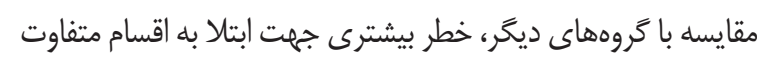

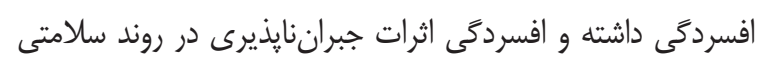

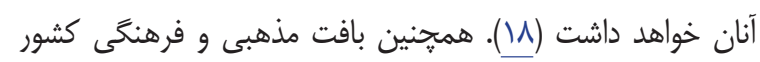

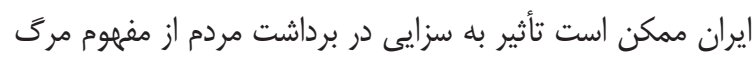

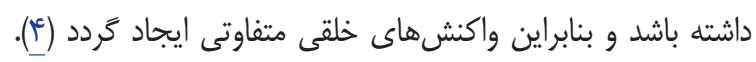

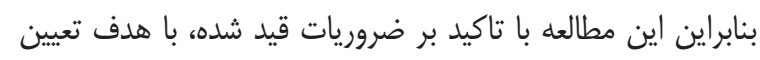

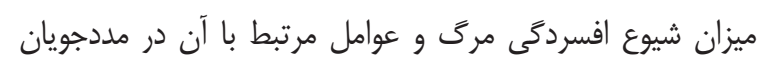

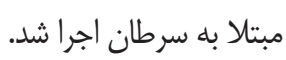

\section{روش مطالعه}

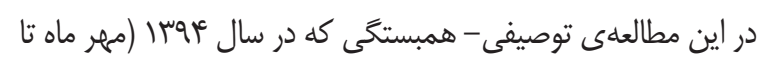

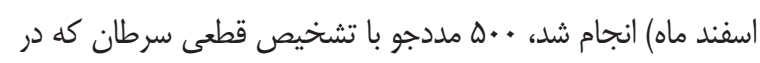

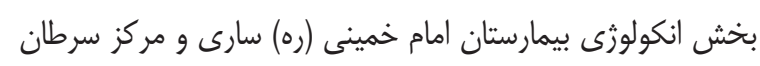

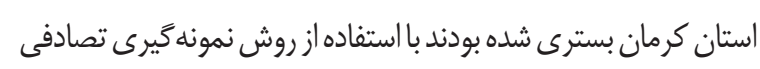

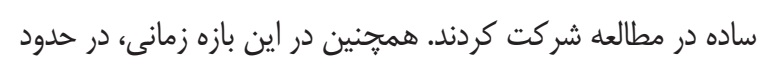

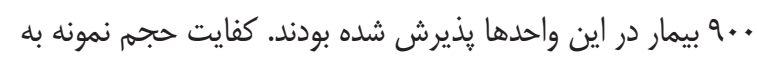

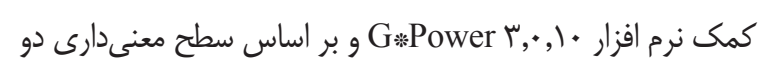

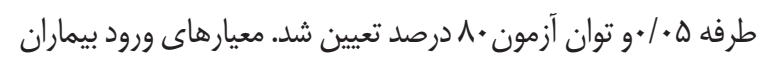

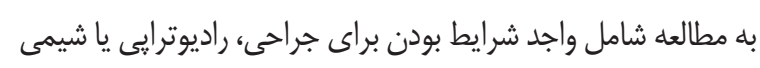

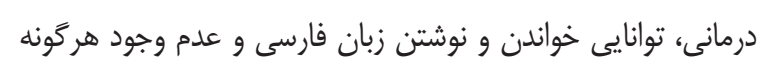

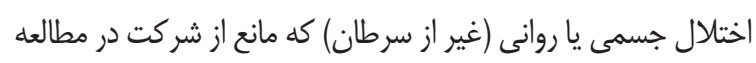

$$
\text { شود (مانند اسكيزوفرنى، دمانس و افسردمى شئ شديد) بود. }
$$

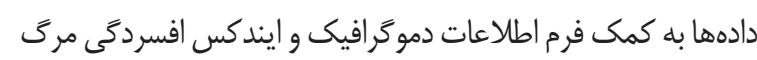

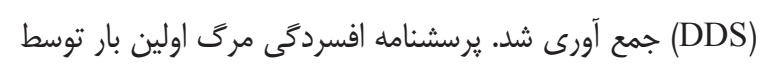
Templer

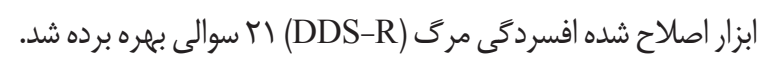

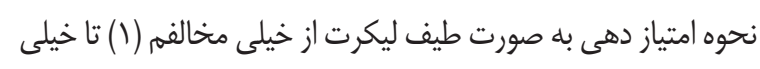

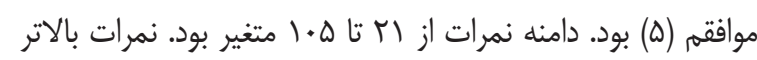

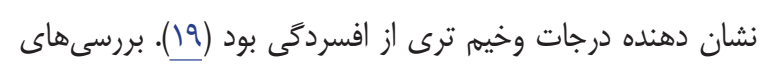

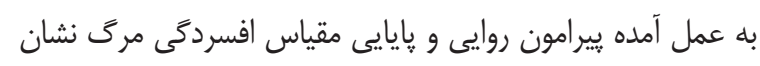

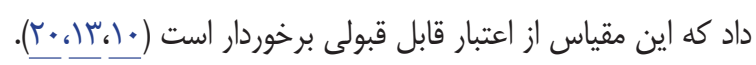
و و همكاران (rempler

\section{مقلمه}

در حال حاضر سرطان به عنوان يك مشكل بزرگ در سلامت افراد

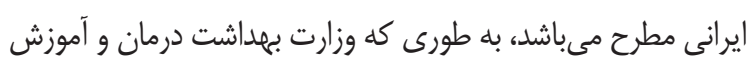

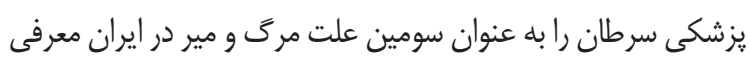

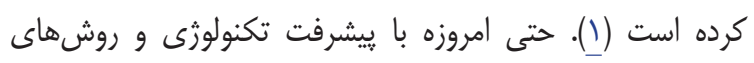

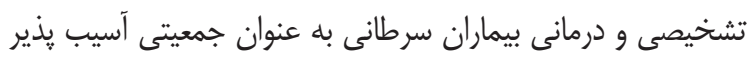

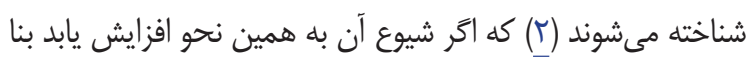

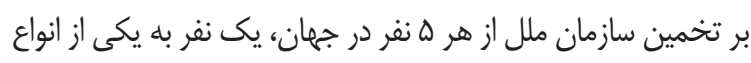

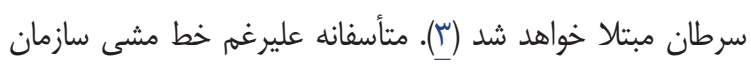

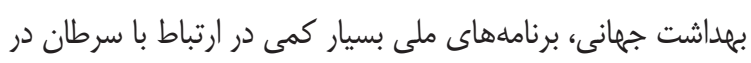

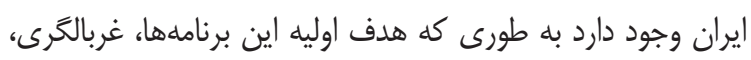

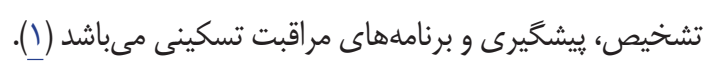

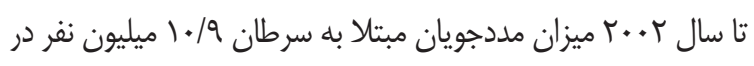

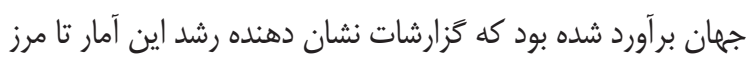

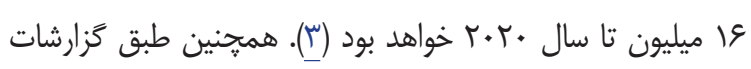

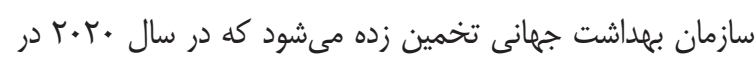

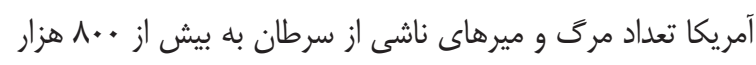

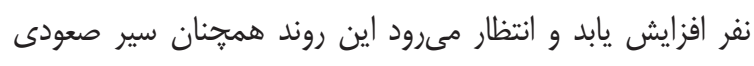

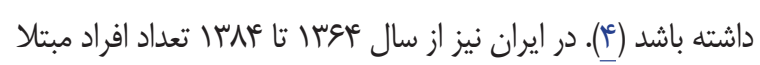

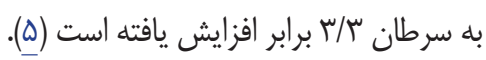

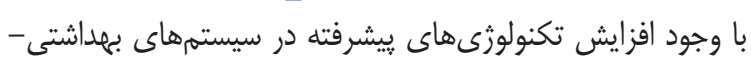

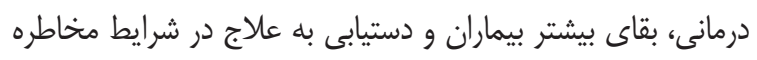

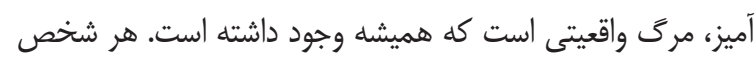

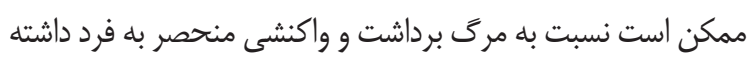

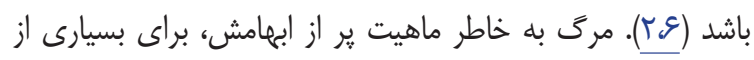

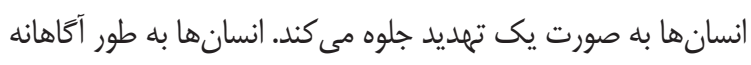

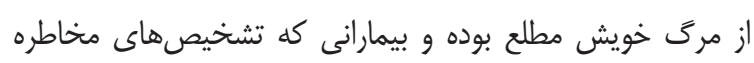

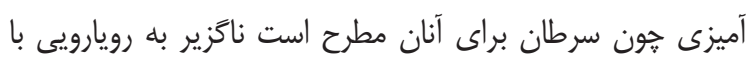

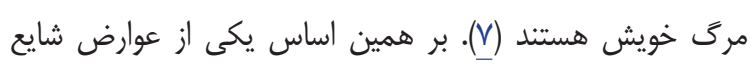

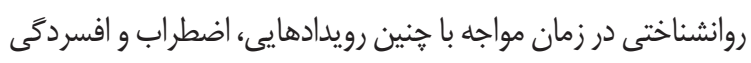

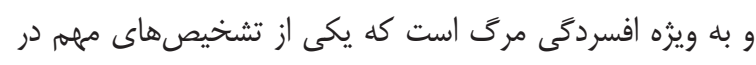
بيماران سرطانى به حساب مى آيد (1).

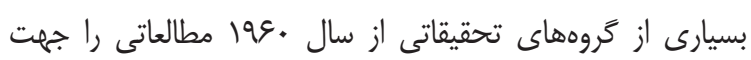

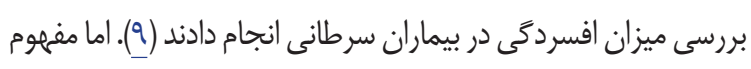

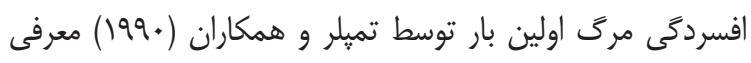

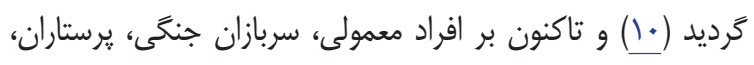

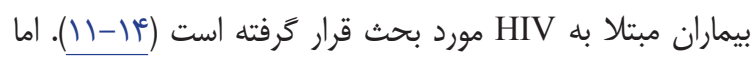

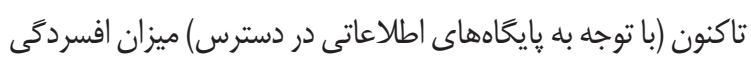

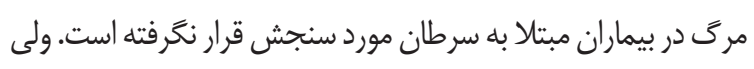
بر اساس مطالعات گَوناكون، ميزان شيوع كلى افسردَّى در بيماران 


\section{امير حسين تَودرزيان و همكاران}

(test استفاده شد. جهت بررسى ييشفرض عدم رابطه هم خطى

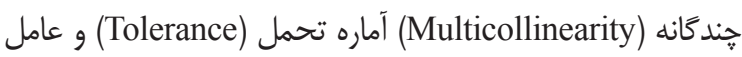
تورم واريانس (Variance inflation factor: VIF) استفاده شدانه

سطح معنى دارى كليه آزمونها كمتر از ه.

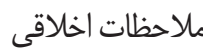

مطالعه حاضر توسط كميته اخلاق دانشكاه علوم يزشكى مازندران مورد تأييد قرار كَرفت (IR.MAZUMS REC.95.106). در ابتدا بيماران در خصوص اهداف و مراحل مطالعه مطلع شدند. همجنين

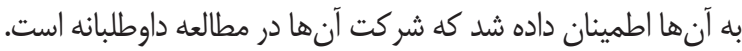
تمامى اطلاعات بيماران با تخصيص كد به هر بيمار، غير قابل فيل

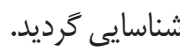

يافته ها

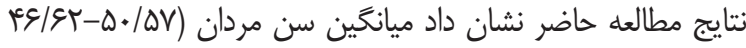
F CI १:

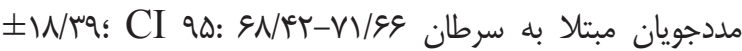

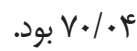

ra/•) مطلوبى را براى اين مقياس گزارش كردهاند (•r). جهت تاييد

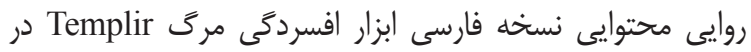

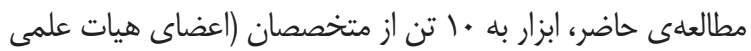
دانشكده يرستارى، روانيزشكان، روانشناسان و اونكولوزيست) داده إنان

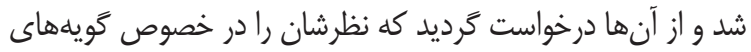

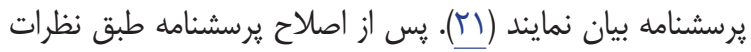

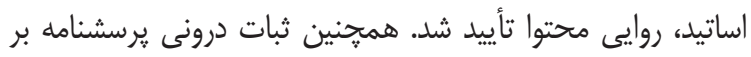
روى مددجويان با سرطان به روش آلفاى كرونباخ 1/ • • محاسبه شد. تجزيه و تحليل داده ها

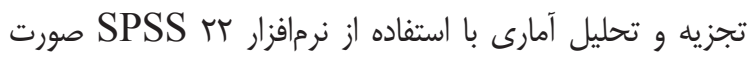
كرفت. از آزمون Kolmogorov-Smirnov جهت برايل براسفى توزيع

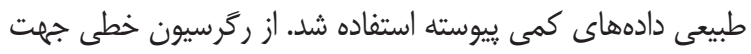

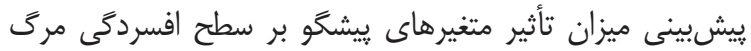

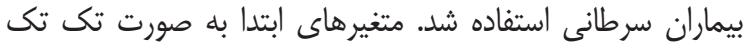
وارد مدل ركرسيونى شدند و سيس به صورت كلى و با روش Enter

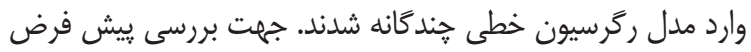
عدم همبستخى باقيماندهها، استقلال خطا، (Independent errors) در ركرّيون خطى، از آزمون دوربين واتسون (Durbin-Watson)

جدول (: ارتباط افسردگى مرگ با متغيرهاى جمعيت شناختى در بيماران سرطانى تحت مطالعه (N=FqV)

\begin{tabular}{|c|c|c|c|}
\hline P value & ميانحين (انحراف معيار) & \multicolumn{2}{|c|}{ متغير ها } \\
\hline$\cdot / T V F$ & $\begin{array}{l}(I V / F V) \vee \cdot / q 1 \\
(1 q / r \Delta) \& q / 1 .\end{array}$ & 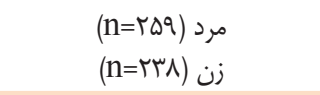 & جنسيت \\
\hline 促 & $\begin{array}{l}(19 / r q) \vee / / r q \\
(1 N / \cdot r) \& q / D .\end{array}$ & 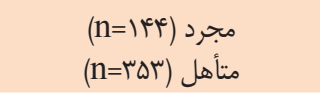 & 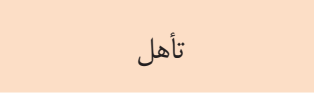 \\
\hline$<\cdot / W r$ & $\begin{array}{l}(19 / \Delta \Delta) V \cdot / \Lambda r \\
(1 N / / F) 9 q / 9 q \\
(I V / T V) \& Q / \Delta .\end{array}$ & 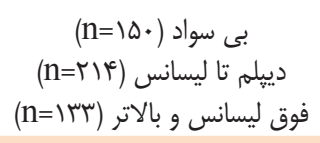 & سطح تحصيلات \\
\hline$<\cdot / . .1$ & 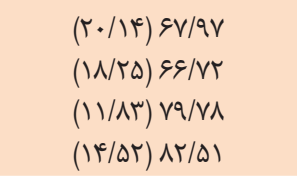 & 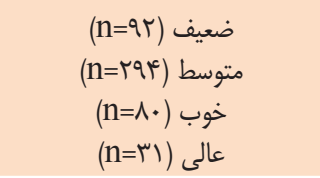 & وضعيت اقتصادى \\
\hline $.1 .+8$ & $\begin{array}{l}(19 / 11) V T / F^{4 F} \\
(1 V / q \&) \& N / q 1\end{array}$ & 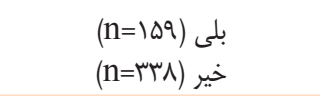 & سابقه مصرف مواد مخدر \\
\hline.$/ 911$ & $\begin{array}{l}(\backslash N / \& \Delta) \vee \cdot / \backslash \Delta \\
(\mid N / T Y) \& Q / ৭ \&\end{array}$ & خلى (n=r/9) & سابقه بيمارى سرطان در خانواده \\
\hline.$|4 V|$ & $\begin{array}{l}(1 N / F r) V \cdot / \& \Lambda \\
(1 N / F \Delta) \& q / F \Lambda\end{array}$ & 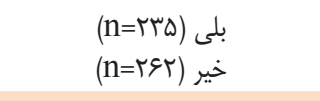 & سابقه بيمارى زمينه اى \\
\hline$<\cdot \mid . .1$ & 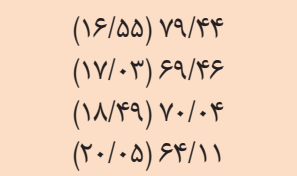 & 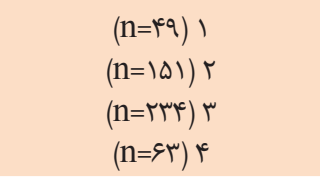 & مرحله سرطان \\
\hline
\end{tabular}


اقتصادى (B=ه/\&V)، نداشتن سابقه مصرف داروهاى ضد افسردگى (B)

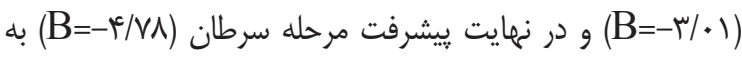

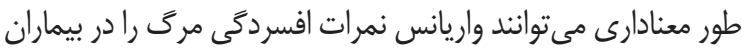

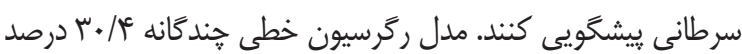
واريانس نمرات افسردگى مرك را تبيين نمود.
نتايج ضريب همبستخى اسيبرمن نشان داد همبستخى معنادارى

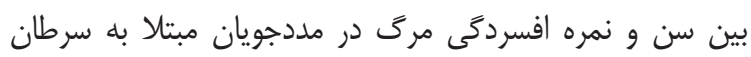

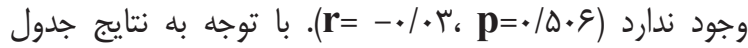

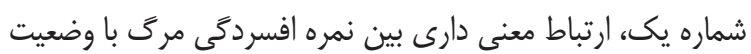

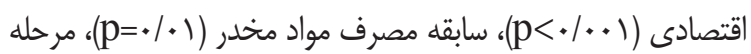

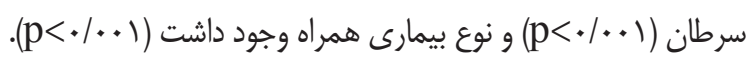
نتايج جدول شماره دو نشان مى دهد تغيير سطح درآمد و وضعيت

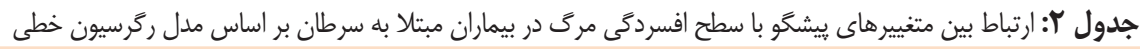

\begin{tabular}{|c|c|c|c|c|c|c|c|c|}
\hline \multicolumn{4}{|c|}{ تعديل شده (جندكانه) } & \multicolumn{4}{|c|}{ تعديل نشده (ساده) } & \multirow{2}{*}{ متغيير ويشكَو } \\
\hline $\mathbf{C I} \%$ १Q & $\mathbf{p}$ & $\beta$ & B & $\mathbf{C I} \%$ $\%$ & p & $\beta$ & B & \\
\hline$r / V \cdot-V / A r$ & $\langle\cdot|\cdot \cdot|$ & $\cdot / F^{2}$ & $\Delta / V Y$ & $r / 9 \uparrow-\Lambda / .9$ & $<\cdot / . .1$ & ./ra & $81 .$. & وضعيت اقتصادى (ضعيف، متوسط، خوب، \\
\hline 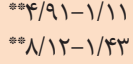 & $\begin{array}{l}. . \cdot t \\
. / .00\end{array}$ & 的 & 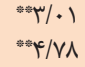 & $\begin{array}{ll}* q / \cdot .-1 / .9 \\
* 00 / / 9-1 / 91\end{array}$ & $\begin{array}{l}<\cdot \kappa q \\
<\cdot / . \cdot 1\end{array}$ & 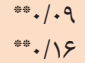 & 每 & سابقه مصرف مواد مخدر (دارد/ ندارد") \\
\hline & & & & & & & & ) جهار) \\
\hline
\end{tabular}

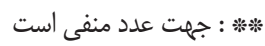

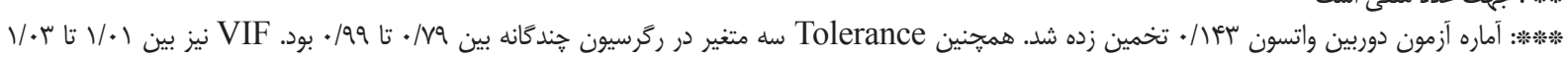
تخمين زده شد.

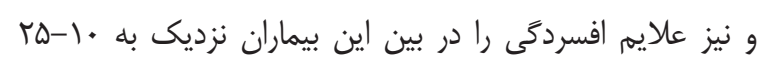

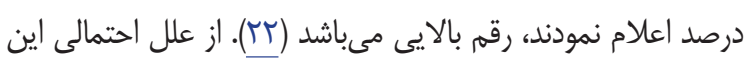

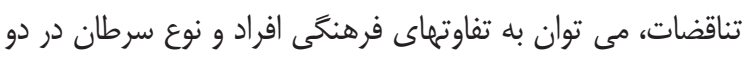

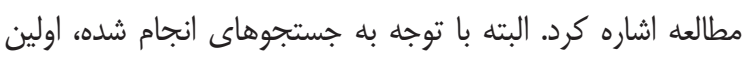

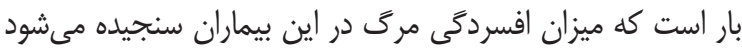

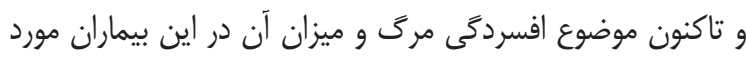

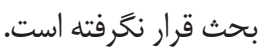
اهميت شناسايى و درمان اختلالات روان شناختى از جمله افسردگى

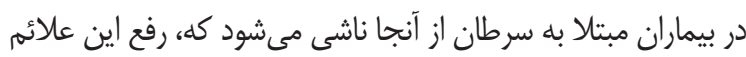

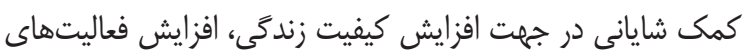

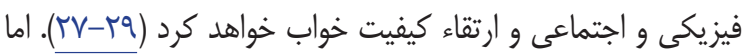

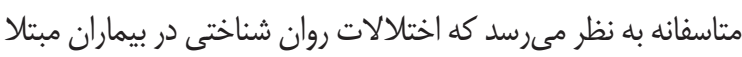
به سرطان اغلب به موقع شناسايى و تشخيص داده نشده و مورد

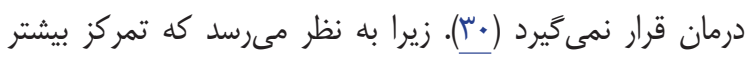
يرسنل درمانى بر مشكلات جسمانى اين بيماران باشد. نتايج يك نيك

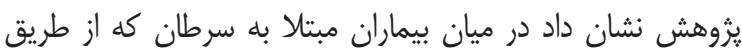

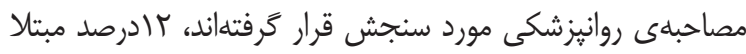

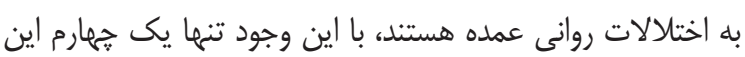

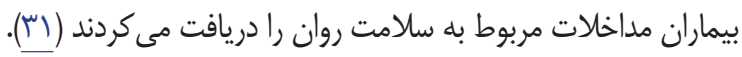

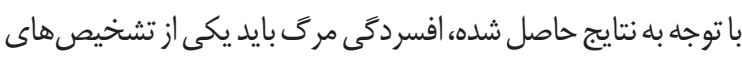

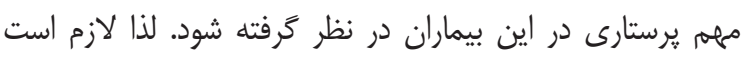

مطالعه حاضر با هدف تعيين ميزان شيوع افسردگى مرى و عوامل

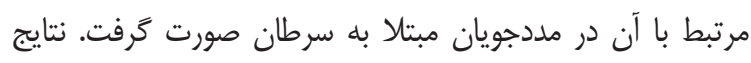

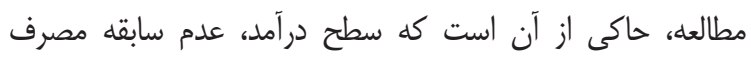

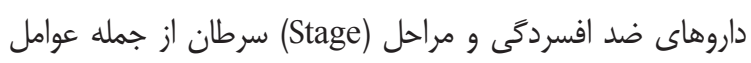

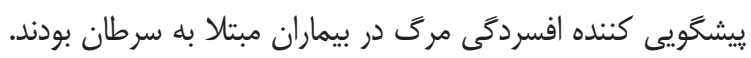

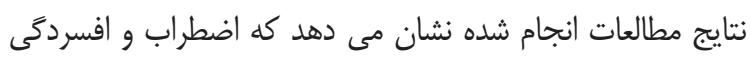

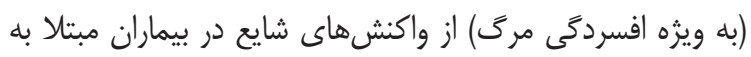

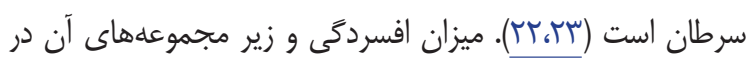
بيماران مبتلا به سرطان طيف گَترده اى را در بر مى گيرد. محققان،

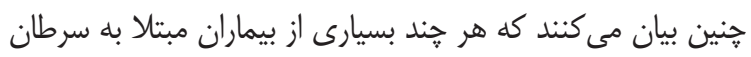

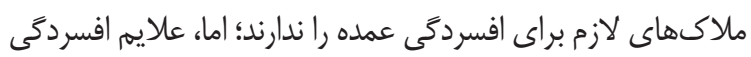
در اين بيماران بسيار شايع است و احتمالاً نياز به درمان دارد (بَآ).

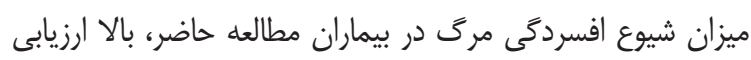

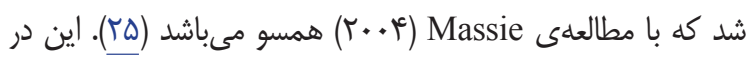

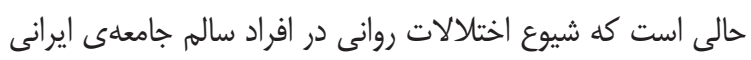

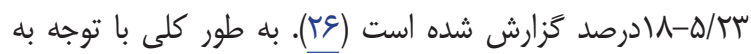

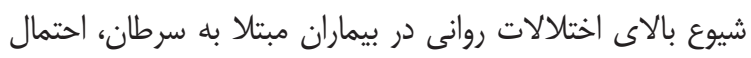
مىرود كه شرايط ويزه بيمارى و فرايند درمان منجر به ايجاد طيف

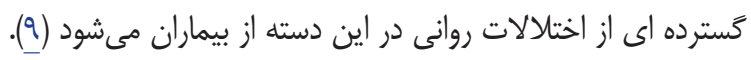

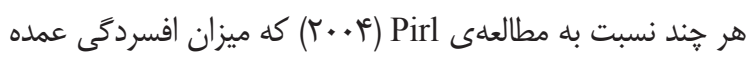




$$
\begin{aligned}
& \text { امير حسين تودرزيان و همكاران } \\
& \text { شيمى درمانى ير رنغتر مىشود. اين در حالى است كه در سيستم }
\end{aligned}
$$

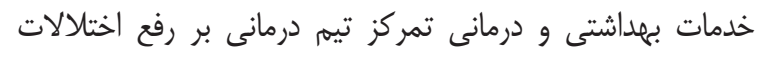

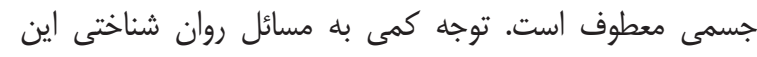

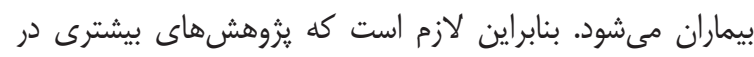

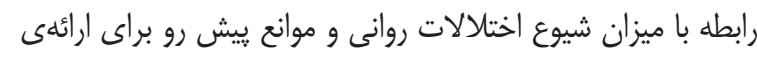

$$
\begin{aligned}
& \text { خدمات روان شناختى مورد نياز در اين بيماران صورت بكيرد. }
\end{aligned}
$$

$$
\begin{aligned}
& \text { تشكر و قدردانى } \\
& \text { از تمامى عزيزانى كه در انجام مطالعه حاضر به تيم تحقيق كمك }
\end{aligned}
$$

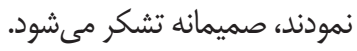

\section{References}

1. Kolahdoozan S, Sadjadi A, Radmard AR, Khademi H. Five common cancers in Iran. Archives of Iranian medicine. 2010;13(2):143.

2. Bahrami N, Moradi M, Soleimani M, Kalantari Z, Hosseini F. Death anxiety and its relationship with quality of life in women with cancer. Iran Journal of Nursing. 2013;26(82):51-61.

3. Eheman C, Henley SJ, Ballard-Barbash R, Jacobs EJ, Schymura MJ, Noone AM, et al. Annual Report to the Nation on the status of cancer, 1975-2008, featuring cancers associated with excess weight and lack of sufficient physical activity. Cancer. 2012;118 (9):2338-66.

4. Sharif Nia H, Ebadi A, Lehto RH, Mousavi B, Peyrovi H, Chan YH. Reliability and validity of the persian version of templer death anxiety scaleextended in veterans of iran-iraq warfare. Iranian journal of psychiatry and behavioral sciences. 2014;8(4):29-37. PubMed PMID: 25798171. Pubmed Central PMCID: PMC4364474.

5. Mousavi SM, Gouya MM, Ramazani R, Davanlou M, Hajsadeghi N, Seddighi Z. Cancer incidence and mortality in Iran. Ann Oncol. 2009;20(3):556-63.

6. Langner T. Choices for living: Coping with fear of dying: Springer; 2002.

7. Emanuel EJ, Fairclough DL, Wolfe P, Emanuel LL. Talking with terminally ill patients and their caregivers about death, dying, and bereavement: is it stressful? Is it helpful? Archives of internal medicine. 2004;164 (18):1999-2004. PubMed PMID: 15477434.

8. Pinquart $\mathrm{M}$, Duberstein PR. Depression and cancer mortality: a meta-analysis. Psychological

$$
\begin{aligned}
& \text { جلسات مشاوره اى در كنار درمانهاى معمول، براى بيماران مبتلا به } \\
& \text { سرطان بركذار شده تا در صورت شناسايى زودرس علائم افسردكى، }
\end{aligned}
$$

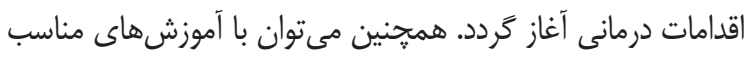

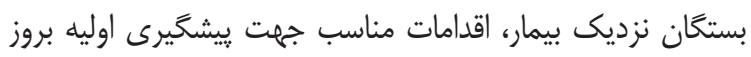

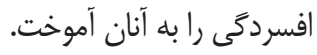

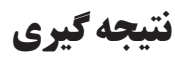

با توجه به فراوانى بالاى افسردگى مرك در بيماران تحت مطالعه،

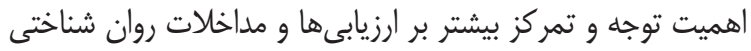

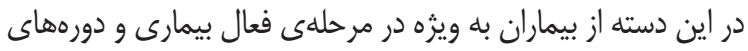

Medicine. 2010;40(11):1797-810.

9. Derogatis LR, Morrow GR, Fetting J, Penman D, Piasetsky S, Schmale AM, et al. The prevalence of psychiatric disorders among cancer patients. JAMA. 1983;249(6):751-7.

10. Templer DI, Lavoie M, Chalgujian H, ThomasDobson S. The measurement of death depression. Journal of Clinical Psychology. 1990;46(6):8349.

11. Tomás-Sábado J, Gómez-Benito J. The Spanish Form of the Death Depression Scale. Perceptual and Motor Skills. 2003;96(1):49-53.

12. Roshdieh S, Templer DI, Cannon WG, Canfield M. The Relationships of Death Anxiety and Death Depression to Religion and Civilian WarRelated Experiences in Iranians. OMEGA Journal of Death and Dying. 1999;38(3):201-10.

13. Hintze J, Templer DI, Cappelletty GG, Frederick W. Death depression and death anxiety in HIVinfected males. Death Studies. 1993;17(4):33341.

14. Alvarado KA, Templer DI, Bresler C, ThomasDobson $\mathrm{S}$. The relationship of religious variables to death depression and death anxiety. Journal of Clinical Psychology. 1995; 51 (2):202-4.

15. Currier MB, Nemeroff CB. Depression as a risk factor for cancer: from pathophysiological advances to treatment implications. Annu Rev Med. 2014;65:203-21.

16. Saracino RM, Weinberger MI, Roth AJ, Hurria A, Nelson CJ. Assessing depression in a geriatric cancer population. Psycho-oncology. 2016. PubMed PMID: 27195436.

17. Mitchell AJ, Chan M, Bhatti H, Halton M, Grassi L, Johansen C, et al. Prevalence of depression, 
anxiety, and adjustment disorder in oncological, haematological, and palliative-care settings: a meta-analysis of 94 interview-based studies. Lancet Oncol. 2011; 12 (2):160-74.

18. Lie HC, Hjermstad MJ, Fayers P, Finset A, Kaasa $\mathrm{S}$, Loge JH. Depression in advanced cancerAssessment challenges and associations with disease load. Journal of Affective Disorders. 2015; 173:176-84.

19. Abdel-Khalek AM. Happiness and death distress: two separate factors. Death Stud. 2005; 29 (10):949-58.

20. Templer DI, Harville M, Hutton S, Underwood R, Tomeo M, Russell M, et al. Death Depression Scale-Revised. OMEGA - Journal of Death and Dying. 2002 March 1, 2002; 44 (2):105-12.

21. Cook DA, Beckman TJ. Current concepts in validity and reliability for psychometric instruments: theory and application. The American journal of medicine. 2006;119(2):166 e7-16. PubMed PMID: 16443422.

22. Pirl WF. Evidence report on the occurrence, assessment, and treatment of depression in cancer patients. Journal of the National Cancer Institute Monographs. 2004 (32):32-9. PubMed PMID: 15263039.

23. Soleimani MA, Lehto RH, Negarandeh R, Bahrami N, Chan YH. Death Anxiety and Quality of Life in Iranian Caregivers of Patients With Cancer. Cancer nursing. 2016.

24. Pasquini M, Biondi M. Depression in cancer patients: a critical review. Clinical Practice and Epidemiology in Mental Health: CP \& EMH. 2007; 3: 2-. PubMed PMID: PMC1797173.

25. Massie MJ. Prevalence of depression in patients with cancer. Journal of the National Cancer Institute Monographs. 2004 (32): 57-71.

26. Ehsanmanesh M. Epidemiological Study of mental disorder in Iran. Journal of Shahrekord University Of Medical Science. 2004; 4:33-42.

27. Weinberger MI, Bruce ML, Roth AJ, Breitbart W, Nelson CJ. Depression and Barriers to Mental Health Care in Older Cancer Patients. International journal of geriatric psychiatry. 2011; 26 (1):21-6.

28. Soleimani MA, Bahrami N, Yaghoobzadeh A, Banihashemi H, Nia HS, Haghdoost AA. Validity and reliability of the persian version of templer death anxiety scale in family caregivers of cancer patients. Iranian Journal of Nursing and Midwifery Research. 2016; 21 (3):284-90.

29. Soleimani MA, Yaghoob Zadeh A, Bahrami N, Sharif SP, Sharif Nia H. Psychometric Evaluation of the Persian Version of the Templer's Death Anxiety Scale in Cancer Patients. Death Studies. 2016:1-11.

30. Zhao L, Li X, Zhang Z, Song C, Guo C, Zhang $\mathrm{Y}$, et al. Prevalence, correlates and recognition of depression in Chinese inpatients with cancer. General Hospital Psychiatry. 2014; 36 (5): 47782.

31. Kadan-Lottick NS, Vanderwerker LC, Block SD, Zhang B, Prigerson HG. Psychiatric disorders and mental health service use in patients with advanced cancer: a report from the coping with cancer study. Cancer. 2005; 104 (12): 2872-81. PubMed PMID: 16284994. Pubmed Central PMCID: PMC1459283. 